\title{
História do Prontuário Médico: Evolução do Prontuário Médico Tradicional ao Prontuário Eletrônico do Paciente - PEP
}

\author{
Medical Records History: Evolution of Traditional Medical Records to Electronic Patient Record - \\ PEP
}

Historia de la Historia Clinica: Evolución de la Historia Clínica Tradicional a la Historia Electrónica del Paciente - HEP

\begin{abstract}
Resumo
O objetivo desta pesquisa é analisar o que se produziu no campo da história do prontuário médico e sua evolução desde o seu surgimento com o prontuário médico tradicional até os dias atuais com o prontuário eletrônico do paciente (PEP) e a sua implantação e utilização, no período de 2010 a 2019, por meio da pesquisa bibliográfica e descritiva, identificando na literatura sobre a história do prontuário médico e sua evolução. Utilizou-se a Biblioteca Virtual de Saúde, base de dados LILACS, e as bibliotecas eletrônicas SciELO. Os descritores utilizados foram "Análise do Prontuário Eletrônico do Paciente (PEP)"; "Pacientes" e "Saúde". Como resultado pode-se observar que a inovação do Prontuário Médico por meio do PEP está intimamente ligada ao envolvimento dos usuários em seu planejamento para o seu sucesso ou fracasso. Conclui-se que este usuário também se preocupa bastante e tem todo o cuidado com o fato de a informática poder ou não eliminar o contato com o paciente.
\end{abstract}

Palavras-chave: Pacientes; Saúde; Prontuário eletrônico.

\begin{abstract}
The objective of this research is to analyze what has been produced in the field of medical record history and its evolution from its emergence with the traditional medical record to the present day with the electronic patient record (PEP) and its implementation and use in the period from 2010 to 2019, through bibliographical and descriptive research, identifying in the literature about the history of the medical record and its evolution. The Virtual Health Library, LILACS database, and SciELO electronic libraries were used. The descriptors used were "Analysis of the Electronic Patient Record (EPR)"; "Patients" and "Health". As a result, it can be observed that the innovation of the Medical Record through the PEP is closely linked to the involvement of users in their planning for their success or failure. It is concluded that this user is also very concerned and is very careful with the fact that information technology may or may not eliminate contact with the patient.
\end{abstract}

Keywords: Patients; Health; Electronic medical records.

\section{Resumen}

El objetivo de esta investigación es analizar lo producido en el campo de la historia de la historia clínica y su evolución desde su surgimiento con la historia clínica tradicional hasta la actualidad con la historia clínica electrónica (EDP) y su implementación y uso en el período de 2010 a 2019, a través de la investigación bibliográfica y descriptiva, identificando en la literatura sobre la historia de la historia clínica y su evolución. Se utilizó la Biblioteca Virtual en Salud, la base de datos LILACS y las bibliotecas electrónicas SciELO. Los descriptores utilizados fueron "Análisis de la historia clínica electrónica del paciente (HCE)"; "Pacientes" y "Salud". Como resultado, se puede observar que la innovación de la Historia Clínica a través del PEP está íntimamente ligada a la implicación de los usuarios en su planificación para su éxito o fracaso. Se concluye que este usuario también está muy preocupado y es muy cuidadoso con el hecho de que las tecnologías de la información pueden o no eliminar el contacto con el paciente.

Palabras clave: Pacientes; Salud; Historia clínica electrónica. 


\section{Introdução}

A necessidade da existência de um documento no qual as informações relativas ao histórico de saúde do indivíduo fossem registradas não é recente. A palavra prontuário origina-se do latim promptuarium e significa "lugar onde são guardadas coisas de que se pode precisar a qualquer momento" ou "manual de informações úteis" ou ainda "ficha que contém os dados pertinentes de uma pessoa" (Houaiss, 2009).

O prontuário é um documento de extrema relevância no atendimento à saúde do paciente, que dispõe da informação fundamental e imprescindível para assegurar a continuidade do tratamento prestado a ele (Santos; Damian, 2017, p. 4). Considera-se importante relatar um pouco da história do Prontuário Médico Tradicional (PMT), traçando uma linha do tempo até os dias atuais.

Massad, Marin; Azevedo (2003) relatam que William Mayo fundou em 1880, junto com um grupo de amigos, a Clínica Mayo em Minnesota nos Estados Unidos, onde foi observado com o passar dos anos, que a maioria dos médicos mantinha o registro de anotações das consultas de todos os pacientes em forma cronológica em um documento único. Fato este, que dificultava o acesso às informações de um determinado paciente. Detectada esta dificuldade, a Clínica Mayo adotou em 1907 um registro individual das informações de cada paciente. As informações passaram a ser arquivadas separadamente e isto possibilitou uma melhor organização e arquivamento dos prontuários. Em 1920 a Clínica Mayo deu consideráveis passos no sentido de padronizar o conteúdo dos prontuários por meio do estabelecimento de um conjunto mínimo de dados a serem registrados.

O processo evoluiu tanto que, desde 1918, praticamente todas as pessoas norte-americanas que tinham sido atendidas em hospitais possuíam um prontuário (Institute of Medicine - IOM, 1991). Os primeiros Prontuários Eletrônicos dos Pacientes (PEPs) surgiram na década de 70, oriundos dos esforços em se estabelecer estruturas mínimas para os registros médicos ambulatoriais (Lovis et al., 2011).

Em 1979, durante a primeira conferência da International Medical Informatics Association (IMIA), os primeiros trabalhos estabeleceram cinco aspectos fundamentais a serem considerados para os sistemas clínicos: ser centrado no paciente, apoiar o uso clínico, promover a educação em tempo real, considerar o fator humano no uso dos computadores e medir o desempenho do uso clínico. Além disso, vinte anos depois, o mesmo instituto norte-americano salientou que: "Para melhorar a Qualidade na Saúde, os profissionais da saúde deveriam interagir efetivamente e eficientemente com os Sistemas de Informação em Saúde - SIS” (Patrício; Maia; Machiavelli; Navaes, 2011).

No início dos anos 90, o governo dos Estados Unidos, por meio do Institute of Medicine (IOM) dos Estados Unidos propôs um estudo com o propósito de definir o que era Prontuário Eletrônico do Paciente (PEP) e também estabelecer melhorias em função das evoluções tecnológicas. Como resultado desse estudo constatou-se que, embora o registro da informação fosse eletrônico, a pobreza do registro comprometia a prestação dos serviços de saúde, pois não oferecia suporte às necessidades dos diversos perfis de consumidores da informação, ou seja, profissionais de saúde, pacientes, administradores e pesquisadores (Patrício; Maia; Machiavelli; Navaes, 2011).

Em 1991, o Institute of Medicine da National Academy of Sciences publicou um estudo intitulado The computer based patient records: an essential technology for health care. Em revisão datada de 1997, o estudo recomendava a adoção do PEP como padrão para os registros médicos e outros relacionados à assistência ao paciente (Patrício; Maia; Machiavelli; Navaes, 2011, pp. 123-124).

O Instituto de Medicina dos Estados Unidos (IOM, 1991) entendeu que o PEP é "um registro eletrônico que reside em um sistema especificamente projetado para apoiar os usuários fornecendo acesso a um completo conjunto de dados corretos, alertas, sistemas de apoio à decisão e outros recursos, como links para bases de conhecimento médico". 
No Brasil, a Sociedade Brasileira de Informática em Saúde (SBIS) reunia profissionais interessados no tema, promovendo encontros anuais entre estes e o Medical Records Institute. As universidades brasileiras que desenvolviam estudos neste setor eram a Escola Paulista de Medicina (EPM), a Universidade de Campinas (UNICAMP) e a Universidade de São Paulo (USP). Os institutos ligados ao Hospital das Clínicas (HC), Instituto do Coração (INCOR) e Instituto da Criança (IC) também desenvolviam projetos pioneiros nesta área. Alguns hospitais, principalmente em São Paulo, Rio de Janeiro e Rio Grande do Sul, discutiram e criaram projetos piloto de implantação dos prontuários eletrônicos.

A informação de registro médico e de enfermagem é considerada relevante diante do contexto de uma unidade de saúde, pois auxilia diretamente no processo de atendimento. Essas informações estão presentes em uma ferramenta chamada Prontuário, que pode ser definido como um catálogo que contém o histórico de atendimento e de saúde do paciente na unidade de saúde (Santos; Damian, 2017, p. 6).

O Prontuário do Paciente é considerado uma ferramenta fundamental para a prestação de serviço em saúde, e que pode ser definido como um registro padronizado e organizado de toda a informação depositada relacionada à saúde de um paciente. Assim, junto com o desenvolvimento dos SIS, dos sistemas hospitalares e dos sistemas administrativos e financeiros, a ideia de um prontuário clínico informatizado se fortaleceu (Patrício; Maia; Machiavelli; Navaes, 2011).

Em 2002 este assunto veio à tona, mostrando que as instituições médicas precisavam melhorar a qualidade de suas informações, para aperfeiçoar recursos e eliminar gastos. Só assim sobreviveriam em um mundo que antes era exclusivo para as indústrias do nosso país. Nesta época no Brasil, os avanços relativos ao PEP estavam restritos às Instituições dos grandes centros e às Universidades. Foi em 2002 que o Ministério da Saúde (MS) propôs um conjunto mínimo de informações sobre o paciente que deveriam constar em um Prontuário Médico (PM) e deliberou sobre o PEP, tornando-se um marco regulatório a ser seguido. Por meio de suas resoluções, o Conselho Federal de Medicina (CFM) reconheceu o PEP como forma legítima de arquivamento das informações do paciente.

Apesar de ser reconhecido pelo CFM por meio das Resoluções 1.638 e 1.639 desde 2002, o PEP ainda não atingiu a maior parte das instituições de saúde do país. E, em várias unidades em que ele é utilizado, a completa substituição do prontuário em papel ainda não ocorreu completamente. Embora a informatização de rotinas administrativas já seja uma realidade nacional, a implantação do PEP ainda enfrenta resistência. O PM é definido pela Resolução n ${ }^{\circ} 1.638 / 2002$, Art. $1^{\circ}$ como sendo um:

Em julho de 2007, o CFM aprovou as normas técnicas para digitalização e uso dos sistemas informatizados para a guarda e manuseio dos prontuários dos pacientes. Enquanto a visão de PM permanece, o seu conceito passa a incluir não somente o documento tradicional em papel, mas também o registro em suporte eletrônico. Portanto, é considerado um documento legal que deve ser preenchido pela equipe multiprofissional de forma íntegra e completa. É um documento de manutenção permanente pelos médicos e estabelecimentos de saúde e pode ser usado posteriormente como prova até que transcorra o prazo de 20 (vinte) anos para efeitos de ações que possam ser impetradas pela justiça. O serviço de arquivo do hospital tem por função guardar tais documentos, por um período mínimo de 20 (vinte) anos, conforme se observa na legislação.

A despeito dos inúmeros avanços pelos quais o PEP passou nos últimos anos e, apesar de todos os investimentos realizados, ainda hoje, muitos profissionais de saúde não utilizam sistemas eletrônicos, porque na maioria dos casos os sistemas não agregam o valor esperado. Estudos concluíram que, apesar de passados trinta anos, os cinco aspectos fundamentais definidos em 1979 no congresso da IMIA continuam muito atuais e deveriam ser perseguidos pela Tecnologia da Informação em Saúde (TIS) no desenvolvimento e implantação dos sistemas (Lovis et al., 2011).

O PM é uma importante ferramenta legal na avaliação da qualidade da assistência prestada ao cliente, pois se trata de um conjunto de documentos padronizados e organizados, destinados ao registro dos cuidados prestados pelos profissionais de 
saúde envolvidos no cuidado e o seu preenchimento incorreto das ações realizadas e registradas no prontuário leva a desassistência por parte da equipe que atua diretamente na prestação do cuidado aos pacientes. (Camargo; Pereira, 2017, pp. 4$5)$.

Nos últimos anos, surgiram novas gerações de sistemas de informação hospitalar que priorizam a integração de dados clínicos e administrativos, com o objetivo de otimizar e qualificar o atendimento, reduzir custos e obter informações relevantes que compõem um perfil da saúde em uma determinada região (Patrício; Maia; Machiavelli e Navaes (2011). Ainda, segundo as mesmas autoras, desde a proposta de implantação do PEP até os dias de hoje, percebe-se uma série de avanços no aperfeiçoamento das tecnologias e a agregação de novas funcionalidades, como a possibilidade de anexar imagens e outros exames complementares, a utilização de sistemas de apoio à decisão e o acesso remoto ao PEP. No entanto, ainda existem muitas dúvidas, críticas e resistência ao uso dos prontuários eletrônicos.

O objetivo deste estudo é descrever a importância e os benefícios da história do PM e sua evolução, desde o seu surgimento com o PMT até os dias atuais com o PEP, com a utilização deste sistema pelas equipes multiprofissionais a fim de estimular o registro sistematizado.

\section{Fundamentação Teórica}

A ideia de se ter um meio onde as informações fossem registradas para poderem auxiliar na continuidade do tratamento, da investigação da doença e no compartilhamento dessas informações, começou com Hipócrates no século V a.C. De acordo com Massad, Marin e Azevedo (2003), o prontuário em suporte de papel vem sendo utilizado há muitos anos. Hipócrates, no século $\mathrm{V}$ a.C. estimulou médicos a fazerem registros escritos, dizendo que o prontuário tinha dois propósitos: (1) refletir de forma exata o curso da doença e (2) indicar as possíveis causas das doenças.

Os autores explicam que o prontuário em suporte de papel já vinha sendo utilizado há muitos anos e revelam, ainda, que Florence Nightingale, precursora da Enfermagem Moderna, quando tratava feridos na Guerra da Crimeia (1853-1856) já relatava que a documentação das informações relativas aos doentes é de fundamental importância para a continuidade dos cuidados ao paciente, principalmente no que se refere à assistência de Enfermagem. É clássica a frase de Nightingale, quando observa a importância dos registros de saúde: "Na tentativa de chegar à verdade, eu tenho buscado em todos os locais, informações; mas, em raras ocasiões eu tenho obtido os registros hospitalares possíveis de serem usados para comparações. Estes registros poderiam nos mostrar como o dinheiro tem sido usado, o que de bom foi realmente feito com ele....'. (Massad, Marin; Azevedo, 2003, p. 2).

O prontuário em papel vem sendo utilizado há muitos anos. Até o início do século XIX, os médicos baseavam suas observações e consequentemente suas anotações, no que ouviam, sentiam e viam e as observações eram registradas em ordem cronológica, estabelecendo assim o chamado prontuário orientado pelo tempo em utilização desde então.

O PEP surgiu não só para substituir o prontuário em papel, mas também para elevar a qualidade da assistência à saúde por meio de novos recursos e aplicações. Novaes (2003, p. 43) chama atenção para o fato de que, ao longo das últimas décadas, os prontuários deixaram de ser denominados "Prontuários Médicos" e passaram para "Prontuários do Paciente". Os prontuários são elementos essenciais para o desenvolvimento das atividades de administração de qualquer unidade hospitalar, para os cuidados e atenção aos pacientes e ainda para subsidiar pesquisas.

"Além dos médicos muitos outros profissionais produzem atualmente registros sobre a atenção que desenvolveram com o paciente, os prontuários tornam-se cada vez mais volumosos, há uma preocupação crescente com a documentação de todos os procedimentos em face a possíveis questionamentos jurídicos, há necessidade de preservação do sigilo das informações registradas, essas têm sido questões rotineiras a serem equacionadas nos serviços de saúde”.(Novaes, 2003, p. 43) 
Conforme Massad, Marin e Azevedo (2003), o PEP é uma forma proposta para unir todos esses diferentes tipos de dados produzidos em variados formatos e épocas diferentes, gerados por diversos profissionais da equipe de saúde e apoio a hospitalidade em distintos locais. Assim, deve ser entendido como sendo a estrutura eletrônica para manutenção de informação sobre o estado de saúde e o cuidado recebido por um indivíduo durante todo seu tempo de vida.

O uso de Tecnologia da Informação (TI) na área de saúde contempla uma multiplicidade de aplicações, desde sistemas típicos de gestão de informações a sistemas de automatização e apoio de tarefas de diagnóstico. Segundo a SBIS, uma aplicação típica e relativamente difundida de gestão de informações é o PEP. Em princípio, esse tipo de sistema de informações auxilia nas tarefas burocráticas e de recuperação de informações de pacientes. O PEP é o processo que incorpora registros de um paciente em um sistema informatizado, com objetivo de gerar informações para diagnóstico médico e para documentação de consultas.

De acordo com o Conselho Federal de Medicina - CFM (2006), os cinco estágios de evolução do registro eletrônico de dados de saúde são: (1) registro médico informatizado paralelo ao registro em papel, (2) registro médico informatizado com foco na redução do volume de papel; (3) registro médico informatizado em nível local envolvendo completa reengenharia dos processos da entidade e incorporação de novas funcionalidades, (4) registro eletrônico de informações da saúde do paciente em nível regional, nacional ou global. Envolve aspectos de interoperabilidade, confidencialidade e segurança de informações e (5) registro eletrônico de dados da saúde com amplas informações do paciente, não exclusivamente ligadas à saúde deste. Visa o acompanhamento integrado do paciente ao longo de toda a sua vida.

Dada a multiplicidade de fatores que podem contribuir para a escolha do paciente de uma clínica hospitalar, Bahadori et al (2016) afirmam que estudar as suas preferências e determinar os fatores que são importantes para ele na sua escolha faz parte dos principais planos das organizações de saúde. Já para a Organização Pan-Americana de Saúde (OPAs), esse tipo de prontuário é uma proposta para atender as demandas dos novos modelos de atenção e de gerenciamento dos serviços de saúde.

Conforme informações do CFM (2006), o PEP corresponde ao terceiro estágio do registro eletrônico de dados, uma vez que suporta a interação da instituição com o paciente, começando com a sua recepção no consultório, ambulatório ou pronto-socorro, até o momento em que é liberado, após o atendimento, assim como os dados históricos de consultas anteriores e resultados de exames, permanecem registrados eletronicamente no sistema (Perez; Zwicker, 2010, pp. 178-179). O prontuário facilita o atendimento médico, pois, entre outras coisas, o paciente não precisa fazer longos relatos de suas consultas e atendimento anteriores.

Em seu artigo intitulado "Preservando a confiabilidade médica na Internet", Sabbatini (2000) relata que com o advento do PEP: "o registro médico deixa de ser um documento passivo, difícil de entender, afastado do paciente, para ser um instrumento ativo, uma central de serviços de informação, um promotor de saúde e de prevenção de problemas, e um educador de pacientes e divulgador de informações confiáveis sobre medicina e saúde".

Os meios de informação podem contribuir de forma positiva para o desenvolvimento da TIC dentro da organização, podendo promover vários tipos de mudanças dentro da empresa, principalmente no que tange a área hospitalar, inclusive quanto aos desafios impostos pelo ambiente de mercado (Goldstein, 2010). Ainda, segundo a mesma autora, acrescenta que a utilização da TIC pode se tornar uma ferramenta estratégica eficaz para prestar informações aos diferentes níveis hierárquicos que compõem o hospital, tanto no processo de planejamento, de operação, de controle das ações estratégicas hospitalares e da tomada de decisão.

O PEP pode ser considerado um sistema poderoso de apoio, para dar suporte ao cuidado à saúde, garantindo a melhora da qualidade de informação, facilitando o acesso aos dados, permitindo a assistência com foco no paciente, disponibilizando seus dados clínicos por meio de registros eletrônicos acessíveis, seguros e altamente úteis. Picchiai e Resky (2018) chamam a atenção sobre a importância da análise financeira e econômica do hospital no sentido de auxiliar a medir sua 
vantagem competitiva, onde a vantagem financeira será realizada por meio dos vários índices calculados com os demonstrativos financeiros da empresa e o desempenho econômico que compara o nível de retorno de uma empresa com o custo de capital, ou seja, a taxa de retorno requerida por seus investidores.

Para Soranz, Pinto e Camacho (2017) a descentralização da gestão para níveis mais próximos do usuário é potencialmente exitosa para o registro de dados clínicos, caso seja feito um adequado monitoramento dos indicadores, auditorias clínicas periódicas e realizado periodicamente informes aos profissionais de saúde com os dados e indicadores acompanhados. Contudo, esse desafio é permanente: com o avanço tecnológico, a necessidade de integração com outros subsistemas se faz necessário; não apenas por uma questão de redução do custo dos pedidos de exames sem evidências científicas sólidas para identificação clínica, mas também para fortalecer a coordenação do cuidado.

\subsection{O PEP - Resolução CFM 1.821/2007 - Mudanças Advindas da Nova Resolução - Aspectos éticos e legais envolvidos no uso do PEP no Brasil}

A Resolução 1.821/2007 do CFM mantém o prazo mínimo de 20 (vinte) anos, a partir do último registro, para a preservação dos prontuários médicos em suporte de papel. Entretanto, autoriza a sua eliminação quando for utilizado sistema informatizado ou quando os prontuários forem digitalizados. Para isso, devem reproduzir todas as informações dos documentos originais, passar por análise obrigatória da Comissão Permanente de Avaliação de Documentos, da unidade médico-hospitalar geradora do arquivo e atender aos requisitos do Nível de Garantia de Segurança 2 (NGS2), das normas constantes no Manual de Certificação para Sistemas de Registro Eletrônico em Saúde (S-RES), elaborado pela SBIS em parceria com o CFM.

As informações encontradas no PEP são fornecidas confidencialmente pelo paciente durante $\mathrm{o}$ atendimento ou obtidas a partir de exames e procedimentos com finalidades diagnósticas ou terapêuticas. Portanto, a confidencialidade das informações do PEP é um direito de todo cidadão, com respaldo na Constituição Federal de 1988. O artigo $5^{\circ}$, inciso X da Constituição garante a inviolabilidade da intimidade, da vida privada, da imagem e da honra das pessoas. Este dever também é previsto no Código Penal Brasileiro, artigo 154, e na maioria dos códigos de ética profissional da saúde.

\subsection{Definição do PEP}

O prontuário do paciente configura-se como "[...] um elemento crucial no atendimento à saúde dos indivíduos, devendo reunir a informação necessária para garantir a continuidade dos tratamentos prestados ao cliente/paciente" (Massad; Marin; Azevedo, 2003, p. 1). Ainda segundo os autores mencionados, o prontuário do paciente tem a finalidade de dar suporte aos processos de atenção à saúde provendo informações médicas; servir como material para pesquisas (tanto no contexto de estudos clínicos quanto no contexto de ensino).

O PEP pode ser definido como um sistema constituído por um banco de dados de informações sobre a vida clínica do paciente, de forma que todos os profissionais possam ter acesso às informações das ações assistenciais prestadas ao paciente e tornar possível um melhor desempenho da atividade clínica, administrativa e de serviços complementares à hospitalidade. Mais do que um sistema de computador o PEP é um processo orientado ao paciente.

Além disso, o prontuário informatizado é considerado não apenas uma ferramenta de registro, mas um facilitador da comunicação e integração dos vários saberes, que auxilia a superar inúmeras dicotomias fundamentadas no modelo biopsicossocial e no fazer interdisciplinar (Moerschberger; Cruz; Langaro, 2017).

\subsection{PEP e a Gestão Hospitalar}

Massad, Marin e Azevedo (2003) entendem que o sistema de PEP foi criado para que médicos e enfermeiros recordassem de forma sistemática dos fatos e eventos clínicos ocorridos em um indivíduo, de forma que os demais 
profissionais da saúde envolvidos no processo de atenção pudessem ter acesso a estas informações. Assim, ele é o mais importante veículo de comunicação entre os membros de uma equipe de saúde responsável pelo atendimento. Além de ser uma ferramenta de trabalho, o PEP fortalece o atendimento integral e ajuda a estabelecer as necessidades do paciente como sujeito singular (Moerschberger; Cruz; Langaro, 2017).

Registros médicos do paciente são essenciais e devem conter todo o histórico de saúde, desde o nascimento até a morte. Além disso, servem de suporte à pesquisa, ao ensino e ao gerenciamento dos serviços de saúde e são também um documento legal dos atos médicos (Goldstein, 2010). Bahadori et al. (2016) afirmam que dada a natureza competitiva do mercado de saúde e a multiplicidade de fatores que podem contribuir par as escolhas do paciente em um hospital, suas necessidades e preferências devem ser consideradas no planejamento e na tomada de decisões de hospitais e de organizações de saúde.

Uma organização hospitalar cria valor tratando a informação, em especial no caso das empresas de serviços. Assim, a informação possui um valor ainda maior, pois contribui para atingir os objetivos da organização. Costa e Portela (2018) destacam que a preocupação com o sigilo e a privacidade das informações armazenadas nos registros está sempre presente no discurso de gestores e profissionais no campo da saúde.

Os PEPs devem ser caracterizados pela simplicidade de utilização, entretanto, à medida que as informações são geradas, a complexidade do sistema aumenta (Patrício; Maia; Machiavelli; Navaes, 2011). Kawakami, Lunardelli e Vechiato (2017) consideram que ao se analisar os conteúdos registrados em PEP, é indiscutível que o seu principal destaque se refere à facilidade de uso no quesito usabilidade, sempre levando em conta os seus aspectos informacionais.

\section{Metodologia}

O presente estudo caracteriza-se como uma pesquisa bibliográfica e descritiva, utilizando-se de procedimentos com embasamento teórico e de eixo documental. Um dos fatores que influenciou o eixo documental da pesquisa é o fato de essa ter como uma de suas características “[...] não exigir contato com os sujeitos da pesquisa. É notório que em muitos casos o contato com os sujeitos é difícil ou até mesmo impossível” (Gil, 2017, p. 46).

Tomando como base os estudos de Gil (2017) e Vergara (2016), a pesquisa pode ser classificada quanto aos fins e quanto aos meios.

Quanto aos fins a pesquisa pode ser classificada como descritiva, já que para Vergara (2016) a pesquisa descritiva expõe características de determinada população ou determinado fenômeno, e pode também estabelecer correlações entre variáveis e definir sua natureza. É uma pesquisa descritiva, de acordo com o critério de classificação proposto por Gil (2017), pois seu objetivo foi o de observar, registar, analisar, classificar e interpretar os fatos sem que haja influência do pesquisador sobre ele.

Quanto aos meios a pesquisa pode ser classificada como bibliográfica. Segundo Vergara (2016), a pesquisa bibliográfica é o estudo sistematizado desenvolvido com base em material publicado em livros, revistas, jornais, redes eletrônicas, isto é, material acessível ao público em geral, podendo ser este fonte primária ou secundária.

Sendo assim, este estudo pode ser classificado como bibliográfico, pois tanto para a elaboração do referencial teórico quanto para a elaboração da metodologia foram utilizados artigos e livros que abordavam o assunto. A questão da dificuldade de contato com o sujeito da pesquisa é certamente um fator delimitador presente neste estudo, uma vez que o acesso à equipe de profissionais que utilizam o PEP, bem como a autorização, por parte de estabelecimentos de Saúde, ao prontuário em si, é embutido de diversos obstáculos que ocorre pelo fato de que, por se tratar de um documento com conteúdos sigilosos, os estabelecimentos apresentam grande resistência e várias restrições com relação a assuntos referentes ao PEP. 
Para a construção do referencial teórico, recorreu-se ao uso de literatura corrente e de referência, tendo sido consultados periódicos científicos (eletrônicos e impressos), livros, manuais e diferentes bases de dados. Foram consultadas tanto publicações nacionais quanto internacionais para atingir o objetivo proposto, com a intenção de identificar o conhecimento constituído sobre a temática da história do PM desde o seu surgimento com o PMT até os dias atuais com o PEP e sua implantação e utilização.

Formulou-se a seguinte temática para o presente estudo: "História do PM desde o seu surgimento com o PMT até os dias atuais com o PE e sua implantação e utilização". A elaboração deste trabalho se realizou por pesquisa em literatura específica a respeito dos temas: PM; PEP; tecnologia de informação na saúde. Foi realizado uma pesquisa documental e bibliográfica no sistema Bireme por meio da Biblioteca Virtual de Saúde (BVS), na base de dados PubMed/Medline, LILACS (Literatura Latino Americana em Ciências de Saúde) e nas bibliotecas eletrônicas SciELO (Scientific Electronic Library Online) e da SBIS (Sociedade Brasileira de Informática em Saúde). Publicações nas línguas portuguesa, inglesa e espanhola foram utilizadas.

Os descritores utilizados para busca das publicações na base LILACS foram os Descritores em Ciências da Saúde (DeCS) "Análise do Prontuário Eletrônico", "Pacientes", "Saúde". Na biblioteca eletrônica SciELO foram utilizadas as seguintes palavras chaves: Análise do Prontuário Eletrônico, Prontuário Eletrônico do Paciente, Informática em Saúde.

A pesquisa foi realizada entre janeiro de 2021 e abril de 2021 onde foram estabelecidos critérios de inclusão: artigos publicados no período de 2010 a 2019, sendo que os critérios de exclusão foram publicações sem data, editoriais, cartas ao leitor e as publicações que tinham um enfoque mais específico em relação ao uso do PEP e patologias. Os artigos foram classificados e, posteriormente, os resultados encontrados foram sintetizados considerando a similaridade de conteúdo.

Cada uma das publicações selecionadas foi investigada em bases de dados disponíveis na Internet. Para melhor estruturar a análise do material pesquisado, foi criado um banco de dados onde os artigos foram listados. No banco de dados, as seguintes variáveis foram identificadas para cada um dos artigos: título, autoria, nome do periódico, ano de publicação, palavra-chave relacionadas.

Uma vez realizado o levantamento do referencial teórico inicial, sucedeu-se, conforme recomendado por Gil (2017), a leitura seletiva e analítica de conteúdos levando-se em conta os objetivos a serem almejados na pesquisa. Por fim, a construção das recomendações de operacionalização aplicáveis ao PEP foi realizada por meio do cruzamento de conteúdos selecionados no referencial teórico.

\section{Discussão}

Assim como o prontuário em suporte tradicional, o PEP apresenta algumas vantagens e inconvenientes. Observa-se que foram apontados vários pontos positivos e negativos na história do PMT ao PEP. É possível destacar a preocupação que o uso da informática não deve eliminar o contato com o paciente. Além do registro documental, o diálogo pessoa a pessoa entre os profissionais facilita o compartilhamento das informações de forma ética, segura e eficaz, ultrapassando o limite imposto pelo espaço virtual e fortalecendo o vínculo entre a equipe (Moerschberger; Cruz; Langaro, 2017).

Muitos médicos reconhecem que, em longo prazo, os registros computadorizados podem melhorar a qualidade do atendimento. Porém, a maioria deles não quer se dedicar ao aprendizado de um novo sistema de trabalho, visto que possuem pouco tempo livre. Além disso, a falta de domínio da tecnologia produz uma sensação de falta de controle e de perda de posição, fazendo com que a pessoa evite contato com o computador. Consequentemente, os sistemas apenas serão úteis caso sejam facilmente entendidos pelos médicos com mínimo esforço para o aprendizado. 
Costa e Portela (2018) afirmam que parte das reclamações de profissionais de saúde sobre o uso do registro eletrônico de saúde seria subterfúgio para encobrir dificuldades com informática e estaria relacionada à preocupação com a perda da autonomia e a pouca importância dada à informação em saúde. Há também a alegação de que o PEP poderia ameaçar a autonomia deste médico por meio de influências e restrições por parte das seguradoras e dos administradores dos estabelecimentos de saúde de posse dessas informações (Health Information Privacy - HIP, 2019).

\subsection{Principais Qualidades das Funcionalidades do PEP}

O PEP é um sistema de armazenamento de informações a que vem substituir o prontuário em papel, o qual tem se tornado insuficiente para atender às necessidades de organização das informações médicas (Massad; Marin; Azevedo, 2003). Há necessidade de integrar os sistemas de informação, de diminuir o retrabalho (digitação após a coleta de dados em fichas escritas) e extinguir o temor da exposição das condutas clínicas no PEP (Rodrigues; Boscarioli; Balloni, 2014).

Galvão e Ricarte (2011, p. 15) indicam o significativo número de pessoas envolvidas em processos relacionados com o PEP ao mencionarem “[...] médicos, enfermeiros, fisioterapeutas, nutricionistas, psicólogos, odontólogos, assistentes sociais [...]" Nesse contexto, é necessário considerar a existência - além das diversas formações profissionais - de outros artefatos como as competências linguísticas, tecnológicas e os fatores culturais que influenciam no comportamento do indivíduo na utilização do PEP (Galvão; Ricarte, 2011).

A capacitação da equipe/usuários é fundamental na operacionalização da informação bem como na sua utilização no processo de tomada de decisão. O papel do gestor é apoiado pelo Gerenciamento de Processos, estudado por Michael Hammer, e que trata de lacunas de desempenho em um determinado período de tempo. Nesse ambiente, o entendimento de excelência operacional, inovação operacional e processos devem ser considerados importantes constituintes da atividade gerencial (Bello; Viltard, 2019).

\subsection{Vantagens do PEP}

Como em toda mudança existem efeitos imediatos que muitas vezes podem não parecer muito positivos, como a necessidade de grande investimento, mas que ao longo do tempo valem o que custaram.

Dentre os pontos positivos está a possibilidade de uma mesma informação ser trabalhada em diferentes partes e, se necessário, reutilizadas, evitando, assim, a redundância informacional (Galvão; Ricarte, 2011), o que não ocorre com as informações registradas em suporte de papel. Além disso, o prontuário em papel “[...] pode apresentar partes ilegíveis ou incompletas, ocupar grande espaço nos arquivos" (Conselho Regional de Medicina do Distrito Federal - CRMDF, 2006, p. 48), problemas esses que podem ser evitados com a utilização do PEP.

Kawakami, Lunardelli e Vechiato (2017) corroboram com o fato de que durante um longo período o prontuário foi utilizado exclusivamente no suporte em papel, mas, com a aquisição de novas tecnologias, houve a possibilidade de transportálo para o formato eletrônico com a existência do PEP. Além disso, o processamento desses dados possibilita que sejam verificadas as tendências sanitárias em determinada população, despertando previamente a atenção dos profissionais e gestores da saúde para possíveis epidemias, assim provocando medidas imediatas de controle (Gonçalves et al., 2013).

\subsection{Desvantagens do PEP}

Por outro lado, é fato que o PEP tem diversos desafios a superar e que hoje certos pontos podem ser considerados como principais desvantagens. O PEP pode apresentar algumas desvantagens, como o fato de depender da disponibilidade de energia elétrica para ser utilizado, enquanto o prontuário em papel não apresenta este fator limitador (CRMDF,2006). 
Conforme Massad, Marin e Azevedo (2003), dentre as desvantagens do prontuário no formato eletrônico temos: necessidade de investimento em hardware, software, dificuldade e resistência dos usuários com os procedimentos informatizados sujeitos a falhas, dificuldade para a completa coleta de dados e constante manutenção, requer treinamentos, investimento em segurança e está sujeito a sabotagens.

Além do alto custo da implantação e a necessidade de treinamento dos profissionais que terão acesso ao prontuário, Gonçalves et al. (2013) também destacam a necessidade de investimento em segurança a fim de garantir o sigilo das informações dos usuários.

\section{Considerações Finais}

Esta pesquisa procurou por meio do levantamento bibliográfico levantar dados sobre a História do PM desde o seu início com a evolução do PMT ao PEP nos dias atuais. As informações sistematizadas e levantadas neste trabalho serão de grande valia aos profissionais de saúde de todos os níveis e teve por objeto difundir uma visão panorâmica e atual onde foram abordados temas de atualidade e de importância para o desenvolvimento, implantação e uso do PEP apresentados no decorrer deste trabalho, assim contribuindo para melhorar a comunicação/informação tanto dos usuários quanto dos colaboradores que é fator essencial em um ambiente hospitalar.

Buscou-se compreender melhor o uso do PEP e sua possível contribuição para o processo de Competência Informacional através do acesso a conhecimento científico atualizado com consequente melhoria na etapa primordial do processo de tomada de decisão; especialmente na saúde pública, ajudando os profissionais a lidar com a imprecisão, na medida em que o conhecimento se consolida e se torna público, pois esta é, de certa maneira, a regra universal contribuindo para a sistematização da estrutura de apresentação da informação médica, presente nos prontuários até hoje.

Por se tratar de uma pesquisa bibliográfica e descritiva, as conclusões obtidas deste trabalho não poderiam ser generalizadas estatisticamente, apenas analiticamente. Contudo, os resultados e as lições aprendidas na pesquisa podem servir para outros pesquisadores, bem como contribuir com a teoria existente.

Ressalta-se a contribuição teórico-reflexiva da pesquisa, evidenciada pelo entendimento de que apesar de o prontuário ainda ser utilizado a maior parte do tempo pela equipe multiprofissional e, principalmente, pelo médico, ele diz respeito ao paciente, portanto, são inúmeras as variáveis internas que podem ser percebidas dentro de um ambiente organizacional hospitalar em conjunto; esta é uma contribuição positiva especialmente na utilização de indicadores para a melhoria de processos, produtos e serviços dentro de uma organização hospitalar, podendo integrar o PEP com as demais estruturas organizacionais a fim de sensibilizar profissionais e gestores resistentes à sua incorporação em serviços de saúde, com o intuito de promover a melhoria da qualidade de assistência ao paciente, pesquisa e gestão.

Percebeu-se que há uma dissociação entre o discurso e a prática no que diz respeito aos registros médicos; é nesse desnivelamento em que a realidade do PEP como elemento acessório e consequente nesse processo, que depende fundamentalmente do compromisso com a qualidade da atenção ao paciente no serviço e a competência profissional. Essa prática pode contribuir para a melhor organização da atenção aos cuidados com o paciente.

Identificou-se a contribuição efetiva do PEP onde os indicadores poderão servir de catalizadores na avaliação de desempenho no que se refere à implantação de um sistema informatizado que possa contribuir para a melhoria de sua eficiência e produtividade, melhorando o desempenho e a qualidade das rotinas em um serviço de saúde por meio do aprendizado tecnológico e para a sistematização de suas ideias, com uma grande variedade de informação e recursos no intuito de tomar a melhor decisão possível. 
Com relação à segurança do prontuário do paciente, seja no suporte tradicional em papel, seja no suporte tecnológico digital, as informações sobre a saúde do paciente são essenciais para garantir uma melhor assistência e prestação de cuidado. O compromisso de todos os profissionais é o de manter os registros dos prontuários sempre legíveis, atualizados, claros e precisos.

Com o avanço científico, novos conhecimentos foram incorporados, aumentando significativamente o volume de informações que no dia a dia são armazenadas sobre o paciente e demais estruturas que envolvem a prestação do cuidado. Nesse processo, o uso de métodos manuais é cada vez mais ineficiente nos serviços de saúde. Importantes informações podem ser perdidas ou, por outro lado, a mesma informação é coletada mais de uma vez por diferentes profissionais da equipe e acaba ficando duplicada no prontuário clínico. Isso colabora para que o custo do atendimento à saúde seja cada vez mais elevado e até mesmo a qualidade seja comprometida por falta, indisponibilidade ou erro no registro dos dados.

Essa é uma das razões pelas quais o computador e todos os demais recursos tecnológicos que apoiam a TI são considerados ferramentas indispensáveis para auxiliar o profissional, garantindo maior agilidade e eficiência na ação. Nesse sentido e como premissa básica, o fluxo de informações pode e deve contribuir sobremaneira para a melhoria da qualidade, da eficiência e da eficácia do atendimento em saúde, e desempenhar as atividades com efetividade e eficiência, facilitando a comunicação e integrando a informação.

Para o sucesso de um projeto na implantação de um PEP, é importante que haja um planejamento prévio, acompanhamento de todas as suas fases e avaliação após implantação para identificar a necessidade de melhorias. Em um país com dimensões continentais e imensa diversidade cultural, o PEP poderá proporcionar mais qualidade ao atendimento e à gestão pública, com condições de superar os desafios para sua implantação e utilização e se tornar uma prática comum na medicina. A implantação dos PEPs em todo o território nacional seria um grande passo em direção à melhoria na qualidade da assistência prestada aos pacientes, por gerar facilidade, agilidade e segurança para os profissionais de saúde. A saúde pública brasileira se tornaria mais completa e precisa.

Em conclusão, é evidente que aprimoramentos técnicos na forma em que os registros são produzidos, processados e armazenados podem contribuir para a sua melhoria, podendo reduzir consideravelmente os problemas com a legibilidade, organização, acesso e utilização dos documentos. A cooperação dos profissionais para a implantação do PEP é imprescindível, já que o processo de integração é longo. Mas, se o objetivo maior é um atendimento de qualidade à saúde da população, as barreiras de resistência devem ser rompidas para prover elementos norteadores que contribuam para a evolução do raciocínio, do desempenho profissional e de pesquisas futuras.

\section{Referências}

Bahadori et al. (2016). Factors contributing towards patient's choice of a hospital clinic from the patients' and managers' perspective. Electronic physician, 8(5), 2378-2387. https://doi.org/10.19082/2378

Bello, H. \& Viltard, L. A. (2019). On Process Management (PM): The apliccability of Michael Hammer's theory in Argentina. Independent Journal of Management \& Production, 10(1), 001-021. https://doi.org/10.14807/ijmp.v10i1.820

Constituição da República Federativa do Brasil. (1988). Promulga a Constituição da República Federativa do Brasil. http://www.planalto.gov.br/ccivil_03/constituicao/douconstituicao88.pdf

Camargo, L. R. L. \& Pereira, G. R. (2017) Análise dos registros realizados pela enfermagem e o possível impacto na auditoria: uma revisão da literatura nacional. Revista de Administração em Saúde, 17(68). http://dx.doi.org/10.23973/ras.68.55.

Costa, J. F. R. \& Portela, M. C. (2018). Percepções de gestores, profissionais e usuários acerca do registro eletrônico de saúde e de aspectos facilitadores e barreiras para a sua implementação. Caderno de Saúde Pública, 34(1), 1-14. https://doi.org/10.1590/0102-311X00187916

Conselho Regional de Medicina do Distrito Federal (CRMDF). (2006). Prontuário médico do paciente: guia para uso prático. Recuperado de: https://www.crmdf.org.br/images/stories/publicacoes/livros/prontuario-medico-do-paciente.pdf 
Galvão, M. C. B., \& Ricarte, I. L. M. (2011). O prontuário eletrônico do paciente no século XXI: contribuições necessárias da ciência da informação. InCID: Revista De Ciência Da Informação E Documentação, 2(2), 77-100. https://doi.org/10.11606/issn.2178-2075.v2i2p77-100

Gil, A. C. (2017). Como elaborar Projetos de Pesquisa. Atlas

Goldstein, M. M. (2010). Health Information Technology and the Idea of Informed Consent. The Journal of Law, Medicine \& Ethics, 38(1), 27-35. https://doi.org/10.1111/j.1748-720X.2010.00463.x

Gonçalves, J. P. P. et al. (2013). Prontuário Eletrônico: uma ferramenta que pode contribuir para a integração das Redes de Atenção à Saúde. Revista Saúde e Debate. 37(96) 43-50 http://dx.doi.org/10.1590/S0103-11042013000100006

Health Information Privacy (HIP). U. S. Department of Health and Human Services. Standards to Protect the Privacy of Personal Health Information. Recuperado de: http://www.hhs.gov/ocr/hipaa/

Houaiss, A., Villar, M.S. \& Franco, F. M. M. Dicionário Houaiss de Língua Portuguesa. Objetiva.

Institute of Medicine - IOM (1991). The Computer-Based Patient Record: Meeting Health Care Needs. The Computer-Basead Patient Record: An Essential Technology for Health Care. Richard, S. D. \& Steen, E. B. (Editors). The National Academy Press, 30-55. https://doi.org/10.17226/18459

Kawakami, T., Lunardelli, R., \& Vechiato, F. (2017). O prontuário eletrônico do paciente na perspectiva das recomendações de usabilidade: proposta de organização da informação. Informação \& Informação, 22(3), 456-483. http://dx.doi.org/10.5433/1981-8920.2017v22n3p456

Lovis et al. (2011). Hospital and health information systems - Current perspectives. Contribution of the IMIA Health Information Systems Working Group. Yearbook of medical informatics, 6(1), 73-82. https://doi.org/10.1055/s-0038-1638741

Massad, Marin \& Azevedo (2003). Prontuário Eletrônico do Paciente: Definições e Conceitos. O prontuário eletrônico do paciente na assistência, informação $e$ conhecimento médico. Massad, E., Marin, H. F. \& Azevedo, R. S. (Editores). São Paulo: USP, 2003. 1-20. Recuperado de: http://www.sbis.org.br/biblioteca_virtual/prontuario.pdf

Moerschberger, M. S., Cruz, F. R. \& Langaro, F. (2017). Reflexões acerca da ética e da qualidade dos registros psicológicos em prontuário eletrônico multiprofissional. Revista da SBPH, 20(2), 89-100. Recuperado de: http://pepsic.bvsalud.org/scielo.php?script=sci_arttext\&pid=S1516$08582017000200006 \& \operatorname{lng}=\mathrm{pt} \& \operatorname{lng}=\mathrm{pt}$

National Academy of Sciences - Institute of Medicina. //https://nam.edu/

Novaes, H. M. D. (2003). A Evolução do Registro Médico. O prontuário eletrônico do paciente na assistência, informação e conhecimento médico. Massad, E., Marin, H. F. e Azevedo, R. S. São Paulo: USP, 2003. 39-46. http://www.sbis.org.br/biblioteca_virtual/prontuario.pdf

Patrício, C. M., Maia, M. M., Machiavelli, J. L. \& Navaes, M. A. (2011). O prontuário eletrônico do paciente no sistema de saúde brasileiro: uma realidade para os médicos? Scientia Medica Porto Alegre, 21(3), 121-131. http://bases.bireme.br/cgibin/wxislind.exe/iah/online/?IsisScript=iah/iah.xis\&src=google\&base=LILACS\&lang=p\&nextAction=lnk\&exprSearch=603941\&indexSearch=ID

Perez, G. \& Zwicker, R. (2010). Fatores determinantes da adoção de sistemas de informação na área de Saúde: um estudo sobre o Prontuário Médico Eletrônico. Revista de Administração Mackenzie, 11, 174-200. https://doi.org/10.1590/S1678-69712010000100008

Picchiai, D. \& Resky, I. (2018). Estudo de Caso de uma pequena clínica: Finanças e Marketing. Revista Sociais e Humanas, 31(2). https://doi.org/10.5902/2317175820604

Resolução $C F M n^{o}$ 1.638. (2002). Define prontuário médico e torna obrigatória a criação da Comissão de Revisão de Prontuários nas instituições de saúde. https://sistemas.cfm.org.br/normas/visualizar/resolucoes/BR/2002/1638

Resolução CFM n 1.639. (2002). Aprova as "Normas Técnicas para o Uso de Sistemas Informatizados para a Guarda e Manuseio do Prontuário Médico", dispõe sobre tempo de guarda dos prontuários, estabelece critérios para certificação dos sistemas de informação e dá outras providências. Brasília, DF. https://sistemas.cfm.org.br/normas/visualizar/resolucoes/BR/2002/1639

Resolução $C F M n^{\circ} 1.821$. (2007). Aprova as normas técnicas concernentes à digitalização e uso dos sistemas informatizados para a guarda e manuseio dos documentos dos prontuários dos pacientes, autorizando a eliminação do papel e a troca de informação identificada em saúde. https://sistemas.cfm.org.br/normas/visualizar/resolucoes/BR/2007/1821

Rodrigues, W. T., Boscarioli, C. \& Balloni, A. J. (2014). A utilização de tecnologias de informação na gestão hospitalar em Cascavel / PR. Por que GESITI? (GEstão de SIstemas e Tecnologias da Informação em Hospitais) - Panorama, Tendências e Perspectivas em Saúde. http://bvsms.saude.gov.br/bvs/publicacoes/por_que_gesiti_gestao_sistemas.pdf >.pp. 357-376

Sabbatini, R. M. E. (2000). Preservando a confiabilidade médica na Internet. Revista Check-up. www.sabbatini.com/renato/papers/checkup-10.htm

Santos, B., \& Damian, I. (2017). Análise da competição de informações por meio de uma transição do prontuário físico ou eletrônico. Revista Cubana de Información em Ciencias de la Salud, 28(4). http://www.acimed.sld.cu/index.php/acimed/article/view/1177/707

Sociedade Brasileira de Informática em Saúde (SBIS). Cartilha sobre Prontuário Eletrônico - A Certificação de Sistemas de Registro Eletrônico de Saúde. (2012). http://www.sbis.org.br/certificacao/Cartilha_SBIS_CFM_Prontuario_Eletronico_fev_2012.pdf

Sociedade Brasileira de Informática em Saúde. Manual de certificação para Sistemas de Registro Eletrônico em Saúde (S-RES) Versão 3.3 Certificação 2009. (2009). Leão, B. F., Costa, C. G. A. C., Silva, M. L. \& Galvão, S. C. (Editores). http://www.sbis.org.br/certificacao/Manual_Certificacao_SBISCFM_2009_v3-3.pdf

Sociedade Brasileira de Informática em Saúde. O que é Informática em Saúde. http://sbis.org.br/o-que-e-informatica-em-saude/ 
Research, Society and Development, v. 10, n. 9, e28510918031, 2021

(CC BY 4.0) | ISSN 2525-3409 | DOI: http://dx.doi.org/10.33448/rsd-v10i9.18031

Soranz, D, Pinto, L. F. \& Camacho, L. A. B. (2017). Análise dos atributos dos cuidados primários em saúde utilizando os prontuários eletrônicos na cidade do Rio de Janeiro. Revista Ciência e Saúde Coletiva [online], 22(3), 819-830. https://doi.org/10.1590/1413-81232017223.33142016

Vergara, S. C. (2016). Projetos e Relatórios de Pesquisa em Administração. Atlas 